\title{
Clinical Features of Chronic Inflammatory Demyelinating Polyneuropathy With Autoimmune Hepatitis
}

\author{
Yi Bao ${ }^{\mathrm{a}, \mathrm{b}}$, Yayong Dinga ${ }^{\mathrm{a}}$, Ran $\mathrm{An}^{\mathrm{a}}$, Xiaodong Liu ${ }^{\mathrm{a}}$, \\ Dianyuzi Xie ${ }^{\mathrm{a}}$, Guangjian $\mathrm{Liu}^{\mathrm{a}}$
}

\begin{abstract}
Chronic inflammatory demyelinating polyneuropathy (CIDP) with autoimmune hepatitis is rare in clinical practice. Here we present a case to describe the relevant clinical features and provide case data for the diagnosis and treatment of the disease. The diagnosis was supported by medical history, physical examination, laboratory and imaging examination, nerve conduction velocity and cerebrospinal fluid examination. The combined disease was identified by autoimmune antibodies and ultrasonography. Combined with the patient's history of chronic progression, clinical manifestations of peripheral nerve damage, cerebrospinal fluid showing protein-cell separation and nerve conduction velocity showing decreased amplitude and conduction velocity, CIDP was confirmed. Combined with the patient's examination result of abdominal bulging, color Doppler ultrasound examination showed cirrhosis, positive $(+)$ anti-liver/kidney microsomal type 1 antibody (anti-LKM-1), considering combination of autoimmune hepatitis type II (AIH-II). The clinical symptoms of both were significantly improved by hormone therapy. CIDP is clinically uncommon in elderly male patients, and it is also rare to have AIH-II. But according to the typical pathogenesis, clinical manifestations, detailed physical examination, laboratory and imaging examination, and neuro-immunological data, clinical diagnosis can be made. Pathological data are needed to confirm the diagnosis. Both have poor natural prognosis. However, the use of hormone therapy according to its pathogenesis can effectively alleviate clinical symptoms and prevent progression.
\end{abstract}

Keywords: CIDP; Autoimmune hepatitis; LKM-1; Cerebrospinal fluid; Cirrhosis

\section{Introduction}

Chronic inflammatory demyelinating polyneuropathy (CIDP)

Manuscript submitted June 2, 2019, accepted July 24, 2019

a Department of Neurology, Taihe Hospital Affiliated to Hubei University of Medicine, Shiyan City, Hubei Province, China

${ }^{b}$ Corresponding Author: Yi Bao, Department of Neurology, Taihe Hospital Affiliated to Hubei University of Medicine, Shiyan City, Hubei Province, China. Email:karlbaoyi@163.com

doi: https://doi.org/10.14740/jnr542 is an immune-mediated peripheral neuropathy, which is mainly seen in adults and common in men. The peak age of onset is $40-60$ years. The onset is more insidious, and there are few pre-infections before the disease $[1,2]$. Clinical manifestations include facial paralysis or ophthalmoplegia, dysarthria, dysphagia, muscle weakness, sensory disturbances, weakened or disappeared tendon reflex, postural hypotension, sphincter dysfunction and arrhythmia. The natural course of disease includes three forms: stepwise progression, steady progression and remission-relapse. Progress lasts from months to years, averaging 3 months. Cerebrospinal fluid (CSF) protein-cell isolation and slow nerve conduction speed can usually be used for clinical diagnosis, and neurological biopsy is required for diagnosis [2-4]. The treatment effect of adrenocortical hormone is good [3-5].

Autoimmune hepatitis (AIH) is a chronic progressive hepatic inflammatory disease mediated by autoimmune response. It occurs mostly in women. The ratio of male to female is $1: 4$, with peak ages of $10-30$ years and over 40 years. It occurs worldwide [6,7], but relatively rare in China. Severe cases can rapidly progress to cirrhosis and liver failure. Most patients show chronic hepatitis without any symptoms. Cirrhosis occurs at the time of visiting a doctor. Some patients with AIH have other common autoimmune diseases such as rheumatoid arthritis, thyroiditis, ulcerative colitis, type 1 diabetes, etc. and even the reason for some patients to see for the first time $[8,9]$. According to serum autoantibodies, AIH can be divided into three types: type I AIH is the most common, with related antibodies being antinuclear antibodies (ANA) and smooth muscle antibodies (SMA); type II AIH is characterized by positive anti-LKM-1; type III AIH is characterized by positive serum anti-soluble liver antigen/liver pancreas (anti-SLA/LP). The prognosis of AIH varies widely. Untreated patients may slowly progress to cirrhosis, or develop into acute, subacute and fullocative liver diseases, and eventually die due to various complications $[6,10]$.

The patient with CIDP described in this article presented mainly with chronic disease, dysarthria, muscle weakness, muscle atrophy, decreased needle sensation like gloves and socks, and skin blackening. Meanwhile, the patient had abdominal distention and positive anti-liver/kidney microsomal type 1 antibody (anti-LKM-1); color Doppler ultrasound suggested cirrhosis, considering the combination of AIH. After hormone therapy, the patient's clinical symptoms improved significantly and cirrhosis was reversed. 


\section{Case Report}

\section{History}

A 67-year-old man with previous physical fitness, presented with numbness and weakness of limbs without obvious inducement 2 years ago which gradually aggravated. At present, the holding was not tight, the walking was unstable, and there was a feeling of stepping on the cotton, accompanied by constipation, no fever and night sweats. After treatment in the local hospital, the patient's symptoms did not improve significantly. In order to seek further treatment, the patient came to our hospital, and the outpatient department received orthopedics. After symptomatic treatment, the patient did not improve significantly and was transferred to the department of neurology after consultation.

\section{Methods}

\section{Physical examination}

Physical examination was conducted on the patient, including vital signs, skin and mucous membrane, heart, lung, abdomen and limbs. Nervous system was examined including brain higher function, cranial nerve, movement, sensation, deep and shallow reflex, pathological sign, ataxia movement and meningeal stimulation sign.

\section{Laboratory examination}

Examination was conducted in the laboratory of our hospital, including blood routine examination, erythrocyte sedimentation rate, vitamin, liver and kidney function, blood glucose, blood lipid, coagulation function, B-type natriuretic peptide (BNP), autoimmunity antibody, tumor marker, tuberculosis antibody, hepatitis marker and thyroid function.

\section{CSF examination}

After informed consent of the patient and family members, the patient was placed on the bed with the curved side, hands clasped the knee, local anesthesia was performed with lidocaine, and the needle was punctured at L3-4. CSF examinations included color, transparency, protein characterization, sugar characterization, cell count and classification, protein quantification, chloride, sugar, lactate dehydrogenase (LDH), high-sensitivity $\mathrm{C}$-reactive protein (hsCRP) and adenosine deaminase (ADA). After the operation, the puncture needle was pulled out and fixed with a sterile gauze block. Pillow was removed and patient lied flat for $6 \mathrm{~h}$.

\section{Electromyography and color Doppler ultrasonography}

In response to the patient's numbness and weakness in the limbs, the nerve conduction velocity and electromyography of the extremities were performed by a highly trained technician in the neurological function room of our hospital. For the abdominal distension of the patient, abdominal color Doppler ultrasonography was performed by a highly trained technician in the color Doppler room of our hospital.

\section{Treatment and follow-up}

After informed consent of the patient and family members, patient was given prednisone $500 \mathrm{mg} /$ day, intravenous infusion, for three consecutive days and then directly oral prednisone $1 \mathrm{mg} / \mathrm{kg} /$ day, maintained for $1 \mathrm{month}$. Patient returned to the hospital for follow-up visit, and the dose was adjusted according to the changes of the condition. At the time of follow-up, blood routine examination, liver and kidney function, nerve conduction velocity of the extremities and abdominal color Doppler ultrasound were performed.

\section{Results}

\section{Physical examination results}

On physical examination, temperature was $37^{\circ} \mathrm{C}$, pulse was 65 beats $/ \mathrm{min}$, respiratory rate was 20 times $/ \mathrm{min}$, and blood pressure was $126 / 79 \mathrm{~mm} \mathrm{Hg}$. Patient was conscious, had unclear speech, and was helped into the ward. He was cooperative in physical examination. There was no yellow staining and bleeding point on the skin and mucous membranes of the whole body, the skin was dark, and there were no enlargement of superficial lymph nodes, middle trachea, and no enlargement of thyroid gland. Symmetrical chest without deformity, steady breathing, clear breathing sounds in both lungs, no dry and wet rales were heard. The heart boundary was not large, and the heart rate was 65 beats/min. The heart rhythm was regular. No pathological murmurs were heard in the auscultation area of each valve. There were abdominal distention, no tenderness and rebound pain. The hard liver could be touched by a horizontal finger under the right costal margin, and the spleen was not touched under the costal margin. The Murphy's sign was negative. There was no tapping pain in the kidney area, muscle atrophy in the limbs, and mild edema in both lower limbs. Nervous system examination showed muscle strength of extremities of proximal level 4 and distal level 3. Pain, temperature, touch, vibration, positional sensation and sputum reflex disappeared. Pathological signs were negative, and meningeal irritation was negative.

\section{Laboratory examination results}

Blood routine showed white blood cell (WBC) of $1.91 \mathrm{G} / \mathrm{L}$, red blood cell (RBC) of $3.67 \mathrm{~T} / \mathrm{L}, \mathrm{Hb}$ of $92 \mathrm{~g} / \mathrm{L}$, hematocrit (HCT) of 0.301 , mean corpuscular hemoglobin $(\mathrm{MCH}) 306 \mathrm{~g} / \mathrm{L}$, red cell distribution width (RDW) of $17.3 \%$, platelets (PLT) of 115 $\mathrm{G} / \mathrm{L}$ and platelet distribution width (PDW) of $11.6 \%$. Blood 
biochemistry showed normal electrolytes, liver function, renal function, blood lipids, blood sugar; albumin (Alb) was 35.5 $\mathrm{g} / \mathrm{L}$. Tumor markers, central nervous system specific protein S100 $\beta$, serum ferritin, alpha-fetoprotein, TB-spot, tuberculosis antibody-IgG, tuberculosis antibody-IgM, human immunodeficiency virus (HIV), hepatitis B virus (HBV), hepatitis C virus (HCV), TP-PA, urine routine and coagulation function were normal. Thyroid function showed thyroid-stimulating hormone (TSH) of $13.258 \mathrm{mU} / \mathrm{L}$, FT3 of $2.51 \mathrm{pmol} / \mathrm{L}, \mathrm{VB} 12$ of $195.76 \mathrm{pg} / \mathrm{mL}$ and erythrocyte sedimentation rate (ESR) of $26 \mathrm{~mm} / \mathrm{h}$; anti-LKM-1 was positive $(+)$, and the remaining was negative. Bone marrow puncture results showed leukopenia.

\section{Imaging examination results}

Cardiac color ultrasound showed degenerative aortic valve with mild to moderate incomplete closure, a small amount of regurgitation in the bicuspid and tricuspid valves, low limit of normal cardiac function, and a small amount of pericardial effusion. Ejection fraction value was $50 \%$. Chest X-ray showed left costal diaphragm angle blunt, a small amount of pleural effusion? Pleural thickening? In magnetic resonance imaging (MRI) of the thoracic vertebra, the thoracic vertebrae signal showed a slight uneven reduction, and the blood system disease was to be discharged. Thoracic disc degeneration was shown. Color ultrasound indicated cirrhosis, pelvic effusion, and abdominal wall swelling.

\section{CSF examination results}

CSF pressure was $120 \mathrm{~mm} \mathrm{H}_{2} \mathrm{O}$. CSF routine showed transparent, no clot, Pandy test positive, total number of cells 0 , colorless. CSF biochemistry showed proline of $0.92 \mathrm{~g} / \mathrm{L}, \mathrm{Cl}$ of $121.1 \mathrm{mmol} / \mathrm{L}$, glutamic acid of $2.03 \mathrm{mmol} / \mathrm{L}, \mathrm{LDH}$ of 33 , hsCRP of $0.9 \mathrm{mg} / \mathrm{L}$ and ADA of 0.78 .

\section{Nerve conduction velocity examination results}

Lower extremity motor nerve conduction showed distal latency extended by more than 50\% from the upper limit of the normal value, motor nerve conduction velocity decreased by more than $30 \%$ from the lower limit of the normal value, and $\mathrm{F}$ wave latency extended by more than $20 \%$ from the upper limit of the normal value. The conduction velocity and amplitude of sensory nerve in lower limbs decreased, suggesting that there was demyelinating lesion in the peripheral nerve and the peripheral nerve was generally damaged.

\section{Treatment and follow-up results}

After hormone treatment, the muscle strength of both lower limbs was restored, the gait was normal, the superficial sensation was more sensitive, the skin was whitened gradually and the abdominal swelling was reduced. After 1 month of dis- charge, the patient returned to the hospital: the skin was white as normal, the abdominal distension disappeared, the muscle tension of the limbs was normal and the shallow feeling was basically normal. The blood routine showed that the anemia was better than before, and the liver color Doppler showed improvement of cirrhosis without pelvic fluid.

\section{Discussion}

Diagnosis criteria of CIDP include symptom progression over 8 weeks, chronic progression or remission-recurrence. The clinical manifestations are limb weakness of different degrees, most of which are symmetrical, and a few are asymmetric. Both proximal and distal limbs can be involved, and the tendon reflex of limbs is reduced or disappeared, accompanied by deep and shallow paresthesia. CSF examination shows protein-cell separation. The results of electrophysiological examination show that nerve conduction velocity is slowed, conduction block or abnormal waveform dispersion. Peripheral neuropathy caused by other reasons is excluded from nerve biopsy; corticosteroid therapy is effective $[1,4,11]$. The patient had a 2-year history of chronic progression and relapsing remission. Symptoms mainly manifested as dysarthria, numbness of limbs, muscle atrophy, low muscle tone, sputum reflex disappeared, decreased acupuncture sensation like gloves and socks, and sphincter dysfunction. CSF showed increased protein and protein-cell separation. Nerve examination showed demyelination, decreased amplitude and decreased conduction velocity. After glucocorticoid treatment, the patient's symptoms were significantly improved, according to which clinical diagnosis could be performed, and a nerve biopsy was required to confirm the diagnosis.

This patient had multiple muscle weakness and peripheral nerve demyelination, which needed to be differentiated from multifocal motor neuropathy (MMN), but the unsupported points were: the patient had hypoesthesia and increased protein, and the hormone treatment effect was good [12]. The peripheral nerve damage of this patient was predominant, which needed to be differentiated from recurrent Guillain-Barre syndrome (GBS), but the unsupported points were: long course of disease, no history of precursor infection, no combination of facial nerve paralysis and respiratory muscle paralysis [13]. The patient had peripheral demyelinating lesions, cirrhosis, hypothyroidism and dark skin. POEMS syndrome was also considered, but the patient's M protein was negative [14]. The patient had decreased peripheral nerve depth and superficial sensation, which needed to be differentiated from paraneoplastic neuropathy. However, the patient's repeated examination showed no tumor, and the tumor markers were negative. The hormone therapy was effective, which was not consistent with the disease [15].

The etiology of CIDP is unclear, and immunotherapy is effective, suggesting that the disease has immune-mediated pathogenesis. Prednisone is currently the preferred drug for drug therapy. The clinical symptoms of the patient improved significantly after hormone therapy, suggesting that immune factors were involved in the pathogenesis. However, the com- 
mon onset age of this disease is 40 - 60 years [1]. The patient could be classified as a late-onset case. In patients with severe disease, intravenous immunoglobulin (IVIG) or plasma exchange therapy can be performed first. IVIG therapy is suitable for patients with severe neurological impairment and rapid progression. IVIG therapy is ineffective for patients with CIDP who have sensory impairment as their primary manifestation. Patients with chronic progressive and recurrent CIDP with demyelinating but axonal denatosis respond well to plasma exchange. The vast majority of patients respond effectively to one of these three methods (first-line treatment). If all three methods are ineffective, immunosuppressive agents can be used as second-line drugs, such as azathioprine, cyclosporine, cyclophosphamide, etc. In addition to drug treatment, adjuvant treatment of large doses of B vitamins and nutritional support, functional exercise, etc., can promote limb rehabilitation and reduce complications $[3,4,16]$. Although the patient had severe motor sensory nerve injury at the time of admission, after informing the patient and his families of the indications and contraindication of the three treatment methods, they decided to use hormone shock therapy after discussion. Immunoglobulin or plasma exchange therapy was not used, but the patient's condition recovered well, suggesting that hormone therapy is sensitive and effective for CIPD.

The patient's abdomen bulged, bloating was obvious and the hard liver could be reached under the right costal margin, which was difficult to explain by CIDP. Color ultrasound showed cirrhosis, pelvic effusion and abdominal wall swelling, and positive (+) anti-LKM-1, in accordance with AIHII $[6,17,18]$. LKM-1 is commonly found in AIH or chronic hepatitis $\mathrm{C}$. The patient was excluded from chronic hepatitis $\mathrm{C}$ and AIH could be considered. The disease is most common in women, with a ratio of male to female of $1: 4$, and there are two peak ages of onset at $10-30$ years and above 40 years $[6,7]$. It is a rare case of an elderly man.

The patient needed to be differentiated from primary biliary cirrhosis in terms of clinical symptoms and laboratory tests, but the patient was an elderly man with no weakness, jaundice or pruritus. Liver function examination showed that alkaline phosphatase and gamma-glutamyl transpeptidase were normal; serum anti-mitochondrial antibody M2-specific antibody was negative and could be identified. However, the shortcoming was that there was no pathological examination. In addition, it needs to be differentiated from primary sclerosing cholangitis, chronic viral hepatitis, alcoholic steatohepatitis and drug-induced liver damage [19, 20].

The clinical features of AIH are increased serum aminotransferase, hypergamma-globulinemia and positive autoantibodies. Severe cases can progress rapidly to cirrhosis and liver failure. Studies have shown that patients with severe AIH have a survival rate of $50 \%$ at 3 years without treatment and $10 \%$ at 5 years. After treatment, the patient's 20-year survival rate reached $80 \%$, and the life expectancy is not significantly different from that of normal healthy people matched by gender and age. Absolute indications for treatment include serum aspartate transaminase (AST) $\geq 10$ times the upper limit of normal, or serum AST $\geq 5$ times the upper limit of normal, and $\gamma$-globulin $\geq 2$ times the upper limit of normal; histological examination showed bridging necrosis or multi-lobular necro- sis $[6,21,22]$. The patient's liver function was normal and no histological examination was performed. However, this patient had relative indications: fatigue, joint pain, jaundice and other symptoms, abnormal serum AST and gamma-globulin levels, which were lower than the absolute criteria. Color ultrasound indicated cirrhosis, and treatment was needed to relieve the symptoms.

The main purpose of AIH treatment is to relieve symptoms, improve liver function and pathological tissue abnormalities, and slow down the progression to liver fibrosis. Glucocorticoid alone or in combination with azathioprine is currently the standard treatment for AIH. This patient had a relative indication of treatment, and because of the combination with CIDP, the preferred treatment for both was hormones. Due to the critical condition, hormone shock therapy and long-term maintenance therapy were adopted. The patient's cirrhosis was improved and the treatment was effective, which supported the diagnosis of AIH-II type.

This case has some highlights and shortcomings. This patient was an elderly man, with late-onset CIDP, with typical peripheral nerve damage in CIDP. At the same time, the disease was combined with AIH-II, which is rare in epidemics, considering the pathogenesis is immune-mediated. The patient had nervous system, digestive system, circulatory system and skin damage. After hormone therapy, he achieved significant improvement, which is the highlight of this case. However, this case is a single-sample clinical case and lacks large sample support. In addition, this case lacked neurological data, no segmental demyelination changes, no reduction in myelinated nerve fibers, insignificant inflammatory response, demyelination and remyelination and other pathological evidence.

\section{Conclusions}

CIDP is uncommon in clinical practice, and it should be considered when there is chronic progression and remission of recurrence, mainly manifested as disarticulation, numbness and weakness of limbs, muscular atrophy, low muscle tension, disappearance of tendon reflex, hypoacusia of gloves and socks, and sphincter dysfunction. The nerve conduction velocity and CSF examination should be improved in time for clinical diagnosis. A neuropathological examination is also needed for the diagnosis. When combined with abdominal distension and liver damage, AIH should also be considered. Timely treatment can relieve symptoms, improve liver function and pathological tissue abnormalities, and slow the progression to liver fibrosis.

\section{Acknowledgments}

We are grateful to the medical staff who have treated those patients carefully.

\section{Financial Disclosure}

This article is a clinical observation study without funding. 


\section{Conflict of Interest}

The authors declare that there is no conflict of interest.

\section{Informed Consent}

At the time of the retrospective analysis carried out here, we no longer had access to the patient.

\section{Author Contributions}

YB, YYD, RA and DYZX: data collection; YB, YYD and $\mathrm{XDL}$ : data analysis; YB and YYD: manuscript preparation; YB and GJL: reviewing of manuscript.

\section{References}

1. Koller H, Kieseier BC, Jander S, Hartung HP. Chronic inflammatory demyelinating polyneuropathy. N Engl J Med. 2005;352(13):1343-1356.

2. Ikeda S, Nishi R, Kawagashira Y, et al. Clinicopathological features of CIDP subtypes. Journal of the Neurological Sciences. 2017;381:465-466.

3. Bunschoten C, Jacobs BC, Van den Bergh PYK, Cornblath DR, van Doorn PA. Progress in diagnosis and treatment of chronic inflammatory demyelinating polyradiculoneuropathy. Lancet Neurol. 2019;18(8):784-794.

4. Dalakas MC. Advances in the diagnosis, pathogenesis and treatment of CIDP. Nat Rev Neurol. 2011;7(9):507517.

5. Wakerley BR, Yuki N. Peripheral neuropathies: Subcutaneous immunoglobulin - the future of CIDP treatment? Nat Rev Neurol. 2018;14(3):130-131.

6. Mieli-Vergani G, Vergani D, Czaja AJ, Manns MP, Krawitt EL, Vierling JM, Lohse AW, et al. Autoimmune hepatitis. Nat Rev Dis Primers. 2018;4:18017.

7. Enomoto H, Nishiguchi S. Similarities and Differences in Autoimmune Hepatitis Epidemiology between East and West: Autoimmune Hepatitis in East Asia, Southeast Asia, and South Asia. Inflamm Intest Dis. 2017;1(4):150158.
8. Pamfil C, Candrea E, Berki E, Popov HI, Radu PI, Rednic S. Primary biliary cirrhosis - autoimmune hepatitis overlap syndrome associated with dermatomyositis, autoimmune thyroiditis and antiphospholipid syndrome. J Gastrointestin Liver Dis. 2015;24(1):101-104.

9. Czaja AJ, Carpenter HA. Autoimmune Hepatitis Overlap Syndromes and Liver Pathology. Gastroenterol Clin North Am. 2017;46(2):345-364.

10. Wang Q, Yang F, Miao Q, Krawitt EL, Gershwin ME, Ma $\mathrm{X}$. The clinical phenotypes of autoimmune hepatitis: A comprehensive review. J Autoimmun. 2016;66:98-107.

11. Allen JA, Lewis RA. CIDP diagnostic pitfalls and perception of treatment benefit. Neurology. 2015;85(6):498504.

12. Van Asseldonk JT, Franssen H, Van den Berg-Vos RM, Wokke JH, Van den Berg LH. Multifocal motor neuropathy. Lancet Neurol. 2005;4(5):309-319.

13. Talukder RK, Sutradhar SR, Rahman KM, Uddin MJ, Akhter H. Guillian-Barre syndrome. Mymensingh Med J. 2011;20(4):748-756.

14. Dispenzieri A. POEMS syndrome: update on diagnosis, risk-stratification, and management. Am J Hematol. 2015;90(10):951-962.

15. Antoine JC, Camdessanche JP. Paraneoplastic neuropathies. Curr Opin Neurol. 2017;30(5):513-520.

16. Querol L, Rojas-Garcia R, Diaz-Manera J, Barcena J, Pardo J, Ortega-Moreno A, Sedano MJ, et al. Rituximab in treatment-resistant CIDP with antibodies against paranodal proteins. Neurol Neuroimmunol Neuroinflamm. 2015;2(5):e149.

17. Krawitt EL. Autoimmune hepatitis. N Engl J Med. 2006;354(1):54-66.

18. Mieli-Vergani G, Vergani D. Autoimmune hepatitis. Nat Rev Gastroenterol Hepatol. 2011;8(6):320-329.

19. Carey EJ, Ali AH, Lindor KD. Primary biliary cirrhosis. Lancet. 2015;386(10003):1565-1575.

20. Hirschfield GM, Karlsen TH, Lindor KD, Adams DH. Primary sclerosing cholangitis. Lancet. 2013;382(9904):1587-1599.

21. Danielle M, Yang Y. Review of autoimmune hepatitis: diagnosis and treatment. Journal of Gastroenterology. 2018;8:57-66.

22. Doycheva I, Watt KD, Gulamhusein AF. Autoimmune hepatitis: Current and future therapeutic options. Liver Int. 2019;39(6):1002-1013. 